Running head: CLASS-DIMENSIONAL STRUCTURE OF PTSD

The Class-Dimensional Structure of PTSD Before and After Deployment to Iraq: Evidence from Direct Comparison of Dimensional, Categorical, and Hybrid Models

Mark D. Kramer ${ }^{1,2,3}$, Paul A. Arbisi ${ }^{2,3,4}$, Paul D. Thuras ${ }^{2,3}$, Robert F. Krueger ${ }^{4}$, Christopher R. Erbes $^{1,2,3}$, Melissa A. Polusny ${ }^{1,2,3}$

${ }^{1}$ Center for Chronic Disease Outcome Research

Minneapolis VA Healthcare System, B9-152

One Veterans Drive

Minneapolis, MN 55417 USA

${ }^{2}$ Minneapolis VA Healthcare System, B68-2

One Veterans Drive

Minneapolis, MN 55417 USA

${ }^{3}$ University of Minnesota - Department of Psychiatry

F282/2A West

2450 Riverside Ave $\mathrm{S}$

Minneapolis, MN 55454 USA

${ }^{4}$ University of Minnesota - Department of Psychology

N218 Elliott Hall

75 E River Rd

Minneapolis, MN 55417 USA

Mark.Kramer@va.gov

Paul.Arbisi@va.gov

Paul.Thuras@va.gov

krueg038@umn.edu

Christopher.Erbes@va.gov

Melissa.Polusny@va.gov 
Author Note

Address correspondence to Mark D. Kramer, Minneapolis VA Healthcare System B68-2, 1 Veterans Drive, Minneapolis, MN 55417. e-mail: Mark.Kramer@va.gov, phone: (612) 4675509.

This research was supported by grants from Minnesota Medical Foundation (3662-922706) and Department of Defense Congressionally Directed Medical Research Program (CDMRP; W81XWH-07-2-003). This material is the result of work supported with resources and the use of facilities at the Minneapolis VA Health Care System, Minneapolis, MN. 


\begin{abstract}
The assumption of specific etiology in posttraumatic stress disorder (PTSD) differentiates the disorder from most other psychiatric conditions. A 'risky test' of the assumption of specific etiology and resultant trauma-related symptom dimensions was conducted through structural modeling of PTSD symptoms in soldiers before $(N=522)$ and after $(n=423)$ a combat deployment to Iraq. If PTSD represents a discrete diagnostic entity that emerges after trauma exposure, we hypothesized either the number of latent classes should increase from pre- to postdeployment or symptom dimensions should qualitatively distinguish affected from unaffected classes following trauma exposure. Comparison of latent structural models revealed best fitting hybrid models for PTSD and depression with strong invariance of symptom dimensions across classes both before and after deployment and only quantitative (i.e., severity) differences between classes. These findings suggest PTSD is generally well-conceptualized as a dimensional syndrome worsened but not necessarily elicited by trauma exposure.
\end{abstract}

Key Words: PTSD, depression, dimensions, classes, structure 


\subsection{The Assumption of Specific Etiology}

PTSD claims a unique status among disorders defined in the Diagnostic and Statistical Manual of Mental Disorders (4 ${ }^{\text {th }}$ ed.; DSM-IV; American Psychiatric Association, 1994; $5^{\text {th }}$ ed.; DSM-5; American Psychiatric Association, 2013) in that the criteria for diagnosis include a specified etiology. DSM-IV Criterion A requires exposure to a traumatic event that both involved the threat of death, serious injury, or a threat to the physical integrity of self or others and a response to the event that included fear, helplessness, or horror. In DSM-5, although the requirement of a specified emotional response to the event is dropped, exposure to a circumscribed class of events remains. Moreover, the concomitant re-experiencing, avoidance/numbing, and hyper-arousal symptoms must both post-date and be directly tied to the traumatic event. Thus, unlike other DSM diagnoses, PTSD both assumes and requires a specific etiology (a specific traumatic event), implying that a distinctive class of events is causally related to a unique class of symptoms. Empirically, identification and inclusion of a necessary etiological criterion for a mental disorder such as PTSD, requires a clearly defined pathogenic pathway between the causative factor and the onset and maintenance of the disorder.

Although exposure to a traumatic event is widely accepted to be a specific etiological factor in the development of PTSD, endorsement of PTSD symptoms occurs in the absence of exposure to traumatic events (Bodkin, Pope, Detke, \& Hudson, 2007; Erwin, Heimberg, Marx, \& Franklin, 2006; Gold, Marx, Soler-Baillo, \& Sloan, 2005; Simons \& Silveira, 1994; Solomon \& Canino, 1990). Given plethora findings suggesting PTSD symptoms are common among nontraumatized populations, the assumption that the disorder is a discrete condition arising following a traumatic event requires further testing. If PTSD is indeed a distinct disorder that emerges after traumatic events, one would expect to find a) a distinct class of individuals 
endorsing the symptoms and $\mathrm{b}$ ) that this class emerges following exposure to traumatic events. While it is impossible to test for such a hypothesis in a classical experiment, if we are able to evaluate PTSD symptoms before and after expected trauma exposure we can test this hypothesis in a naturalistic experiment.

\subsection{The Assumption of Structure}

The construct validity of PTSD as a diagnostic entity rests on the assumptions of specific etiology and a causal relationship between the etiological trauma and the multi-factorial symptom presentation. These assumptions, in turn, have implications for the expected underlying structure of PTSD symptoms. The illness is the conjunction of its etiology and pathology. As such, the assumption of specific etiology would appear to qualitatively distinguish between individuals exposed to a qualifying traumatic event from those who have not been exposed to a qualifying traumatic event (i.e., experienced Criterion A vs did not). The assumption of a multi-faceted structure of the symptoms of PTSD would appear to quantitatively distinguish pathological from non-pathological responses to traumatic events (i.e., allowing for differential expression of the syndrome across facets). If the assumption of specific etiology presupposes that PTSD represents a difference in kind from normative stress reactions to adverse events, and the multi-faceted structure presupposes that PTSD represents a difference in degree distinguishing these pathological responses from normative ones, do these assumptions conflict? Would the syndrome best be conceptualized as dimensional, categorical, or some combination thereof? To address this question, investigations of the structure of PTSD have been conducted using each of these conceptualizations.

\subsubsection{Dimensional Conceptualizations of PTSD}


Assuming a dimensional structure, latent trait modeling (LTM) of the 17 DSM-IV PTSD has been conducted using factor analysis. Comparison of models across various measures of PTSD has generally converged on a four or five-factor structure. The 'numbing' model (King, Leskin, King, \& Weathers, 1998) is consistent with DSM-IV PTSD criteria but for separation of criterion $\mathrm{C}$ into avoidance and emotional numbing factors, and the 'dysphoria' model (Simms, Watson, \& Doebbeling, 2002) includes a dysphoria factor comprising the first five Criterion C symptoms and the first three Criterion D symptoms, along with a two-item avoidance factor. The four-factor 'dysphoria' model (Simms et al., 2002) has received additional support through evidence of measurement invariance (Elhai et al., 2009; Meis, Erbes, Kaler, Arbisi, \& Polusny, 2011), literature review (Elhai \& Palmieri, 2011), meta-analysis (Yufik \& Simms, 2010), and external validation (Gootzeit \& Markon, 2011). The five-factor 'dysphoric arousal' model, parsing dysphoria symptoms into an additional factor, has received empirical support as well (Elhai et al., 2011).

\subsubsection{Categorical Conceptualizations of PTSD}

Assuming a categorical latent structure, latent class modeling (LCM) has been conducted in a variety of samples where varying proportions of members were exposed to trauma. In two large, community-based samples of individuals exposed to trauma, Breslau, Reboussin, Anthony, and Storr (2005) found evidence of three latent classes associated with no disturbance, intermediate disturbance, and pervasive disturbance. In both samples, the emotional numbing cluster of symptoms was shown to differentiate no disturbance and intermediate disturbance from pervasive disturbance classes. In the National Vietnam Readjustment Study, latent class analysis was conducted on a sample of individuals evidencing current PTSD symptomatology as defined by the DSM-III-R (American Psychiatric Association, 1987) criteria (Steenkamp et al., 
2012). No disturbance, intermediate disturbance, and pervasive disturbance classes emerged, with avoidance and hypervigilance symptoms differentiating the pervasive from classes of lesser severity. Latent class analyses were conducted on PTSD symptoms in the National Survey of Adolescents - Replication study at two time points following exposure on those reporting prior trauma at Wave 1 (Ayer et al., 2011). Again, three latent classes at both Waves 1 and 2 were identified, no disturbance, intermediate disturbance, and pervasive disturbance classes. The pervasive disturbance class was best distinguished from the intermediate disturbance class by emotional numbing and hyperarousal symptoms at Wave 1, and by re-experiencing, avoidance, and hyperarousal symptoms at Wave 2. To date, then, latent class analyses have suggested some possible differences in kind for PTSD symptoms. However, latent class analyses in these trauma-exposed samples were consistent in identifying three classes differentiated by severity, which may not be inconsistent with dimensional conceptualizations as continuous differences in severity can only be described in terms of alternative classes using LCM. Indeed, when modeling PTSD with comorbid depression and generalized anxiety disorder, three classes also emerge based on severity levels rather than symptom type (Armour et al., 2015; Contractor et al., 2015).

\subsubsection{Dimensional, Categorical, or Mixture Conceptualizations of PTSD}

Two types of analytical approaches have been implemented to compare dimensional, categorical representations, and/or a mixture of the two: taxometric analyses and factor mixture models. Taxometric analytic tools were developed specifically to evaluate evidence for the hypothesis that a taxon (a discrete latent group) may be identified by a specific set of indicator variables (e.g., Meehl, 1995; Waller \& Meehl, 1998). Taxometric analysis is conceptually similar to two-class latent class analysis, though the statistical methods developed under this 
rubric are distinctive from those employed in latent class analysis. A taxon is not synonymous with a latent class, as Meehl (1992) states 'All taxa are classes, but not all classes are taxa... Perhaps the commonest way of explicating the taxon concept is to say that a genuine taxon is a natural kind, as opposed to an arbitrary class.' Ruscio, Ruscio, and Keane (2002) performed three taxometric procedures using interview and self-report data in a sample of male, treatmentseeking combat veterans. Each procedure yielded results suggesting that posttraumatic stress responses are dimensional in nature. These results were replicated using interview and selfreport data in a male, community-based sample of male combat veterans with a lower estimated prevalence of PTSD (Forbes, Haslam, Williams, \& Creamer, 2005). The results of these analyses suggest that PTSD symptomatology is dimension-like, that no taxa or 'natural kind' emerges when dimensional and categorical representations of the syndrome are directly compared.

To our knowledge, few studies to date have used factor mixture modeling in evaluating the structure of PTSD. Factor mixture modeling provides for simultaneous inclusion of both latent classes and multiple latent dimensions within the same structural domain (Lubke \& Muthén, 2005). The focus of extant studies, however, has been largely to compare the two predominant LTMs of DSM-IV PTSD symptomatology, namely the four-factor 'numbing' (King et al., 1998) and 'dysphoria' (Simms et al., 2002) models. In a Canadian sample of veterans referred for psychiatric evaluation, Naifeh, Richardson, Del Ben, and Elhai (2010), used a factor mixture modeling approach specified to identify one- or two-class variants of four-factor models parameterized as the 'dysphoria' and 'numbing' models. Two-class, four-factor solutions fit better than one-class, four-factor solutions in both cases. The differences in factor means between class one and class two were interpreted as differences in severity. Shevlin and Elklit 
(2012) used confirmatory factor mixture modeling to compare the fit of the 'numbing' and 'dysphoria' models in a combined sample of subpopulations exposed to different types of trauma. A two-class model in which the factor structures of the 'numbing' and 'dysphoria' models were parameterized to distinguish classes were compared with one- and two-class 'dysphoria' and 'numbing' factor mixture models that varied across class in item thresholds (i.e., item endorsement differences across class). The best-fitting model was the former, with class membership and therefore the preference for the dysphoria or numbing model of PTSD associated with trauma type. Unfortunately, the question of whether the classes reflected differences in kind versus degree/severity was not adequately addressed in either study as the focus was on comparison of the two models. The first study found differences in severity in the two classes, and the latter did not report models with alternative measurement invariance constraints.

Taken together, the majority of the work on the latent structure of PTSD has utilized one analytical approach, usually a dimensional one. In so doing, the resulting underlying structure of the syndrome is preordained: the structure is dimensional (four factors), categorical (three classes), or a class-dimensional hybrid (two classes, four factors). The analytic approach has dictated the structure, and in the latter approaches the classes are largely differentiated by severity. Taxometric findings are limited in being specifically designed to test the taxonic conjecture, i.e., the evidence for a latent structure consisting of a taxon group and its complement group, and are not designed to parameterize other alternatives (e.g., a potential multi-dimensional structure of the symptoms, or more than two latent groups). If PTSD symptoms represent a number of dimensions that vary continuously in the population, perhaps regardless of trauma exposure (e.g., Gold et al., 2005), then we have defined the syndrome based on arbitrary cut 
points of severity on dimensions that may or may not be necessitated by trauma. To our knowledge, there have been few 'risky tests' of the assumption that the symptom dimensions of PTSD categorically differentiate pathological from normative responses to Criterion A events rather than differentiating the degree of response to stressful events.

\subsection{The Current Study: Direct Comparison of Latent Trait, Latent Class, and Factor}

\section{Mixture Models}

Trauma exposure cannot be manipulated in the laboratory to experimentally test the etiological hypothesis or to document the sequelae of various forms of trauma. The rationally derived criteria appear to reflect stress reaction dimensions that the majority of the literature suggests quantitatively distinguish debilitating from lesser degrees of distress. Evidence supporting qualitative distinctions in endorsement of PTSD criteria between affected versus unaffected and exposed versus unexposed individuals is limited. The current study is one of few that assessed individuals prior to exposure to potentially traumatic combat-related events (Polusny et al., 2011). Using a quasi-experimental design, we tested the hypothesis that PTSD criteria will qualitatively distinguish between pathological and normative stress responses following trauma exposure through comparison of latent trait models, latent class models, and factor mixture models. PTSD symptomatology was assessed in a sample of National Guard Soldiers both before and after deployment to Iraq. Evidence for qualitative differences in the latent structure of PTSD could arise following deployment in a number of ways: 1) a latent PTSD class not present prior to combat exposure should emerge following combat exposure, 2) the symptom facets should qualitatively distinguish affected from unaffected classes following combat-related trauma exposure, or 3) the symptoms may be expected to differ in the manner in which they coalesce (i.e., in the number and/or nature of the factors) before and after trauma 
exposure. In addition, we examined the specificity of PTSD structural differences before and after trauma by comparing latent trait models, latent class models, and factor mixture models in depression, a disorder that does not assume the specific etiology of trauma exposure or a multidimensional structure causally-linked to trauma. Lacking a qualitative etiological delineation between those with and without depression, structural differences before and after trauma exposure may be expected to be quantitative.

\section{Material and Methods}

\subsection{Participants}

The sample was recruited from a US National Guard Brigade Combat Team (BCT) deployed to Iraq during Operation Iraqi Freedom (OIF) from March 2006 through July 2007. During mobilization training, participants $(\mathrm{N}=522)$ were asked to complete a self-report survey comprising a battery of risk and resilience measures. Administration of the surveys was standardized in a group classroom setting approximately one month prior to deployment. Two to three months after the soldiers returned from deployment, surveys were mailed to participants with a monetary incentive. Response rates were maximized through repeated mailing procedures ( $\mathrm{n}=423 ; 81.2 \%$ of the pre-deployment sample). The assessment battery and description of differences between post-deployment responders and non-responders on pre-deployment variables are described in Polusny et al. (2011), which additionally lists the prevalence of combat-related stressors reported by the soldiers (see Table 2). The sample was predominantly male $(n=462,88.5 \%)$ and Caucasian $(n=490,93.9 \%)$ with $14.2(2.00)$ years of education. Income was not directly assessed. Study procedures were approved by the human subject research review boards of the Minneapolis VA, University of Minnesota, Army, and Army National Guard commands. 


\subsection{Measures}

\subsubsection{Posttraumatic Stress Disorder Checklist (PCL)}

The PTSD Checklist (PCL; Weathers et al., 1993) is a 17-item instrument designed to assess symptoms of PTSD defined in the DSM-IV-TR. Two versions of the PCL were administered to participants, the PCL-C (Civilian) and the PCL-M (Military) during pre- and post-deployment waves of data collection, respectively. The PCL-C references symptoms to the problems and complaints individuals sometimes have in response to 'stressful experiences' and the PCL-M references symptoms in response to 'stressful military experiences.' PCL items were scored on a five-point scale according to how distressing specific problems had been during the prior month (i.e., Not at all, A little bit, Moderately, Quite a bit, Extremely). Average pre- and post-deployment scores on the PCL-C was 26.2 (10.01) and 35.7 (13.96) for the PCL-M, and Cronbach's $\alpha$ were .92 and .94 , respectively.

\subsubsection{Beck Depression Inventory-II (BDI-II)}

The Beck Depression Inventory-II (BDI-II; Beck, Steer, \& Brown, 1996) is a 21-item instrument developed to assess the criteria of depression as defined in the DSM-IV. Each item lists four statements of increasing severity for the assessment of both the presence and intensity of depression symptoms, scored from zero to three. Psychometric properties of the BDI-II are well established (e.g., Arnau, Meagher, Norris, \& Bramson, 2001; Beck et al., 1996; Segal, Coolidge, Cahill, \& O’Riley, 2008). Average pre- and post-deployment scores on the BDI-II were 6.0 (6.82) and $9.7(8.19)$, and Cronbach's $\alpha$ were .91 and .92 , respectively.

\subsection{Statistical Analyses}

The symptom response patterns of PTSD and depression as measured by the PCL and BDI-II, respectively, were examined using latent trait models (LTMs), latent class models 
(LCMs), and hybrid LTM/LCA factor mixture models (FMMs). LTMs represent patterns of symptom endorsement in terms of unobserved trait-dimensions or continua reflecting quantitative differences in liability. LCMs represent patterns of symptom endorsement in terms of unobserved categories or classes of individuals reflecting qualitative differences in liability. Finally, hybrid LTM/LCA FMMs represent patterns of symptom endorsement in terms of both latent trait-dimensions and classes, with class composition representing either quantitative or qualitative differences in liability.

\subsubsection{Latent Trait/Quantitative Models of Liability}

Confirmatory factor analysis (CFA) models both the number of latent dimensions underlying covariation between measured variables and the nature of the factors, or the specific items loading on particular factors. Factors of a confirmatory model may be conceptualized as continuous latent traits accounting for individual differences in the likelihood of endorsement of items/symptoms, or underlying liability dimensions. The structural models of PTSD in the current analyses were informed by prior work with the PCL. Specifically, we compared one-, three-, and two distinct four-factor models ('numbing' model; King et al., 1998; 'dysphoria' model; Simms et al., 2002). One-factor models of depression were compared with two-factor models informed by exploratory factor analyses (EFA) and previous work on the structure of the BDI-II (e.g., Beck et al., 1996; Dozois, Dobson, \& Ahnberg, 1998; Shafer, 2006).

Latent class factor analysis (LCFA) is a non-parametric latent trait modeling approach to representation of a non-normal factor distribution. Here item thresholds and factor loadings are held invariant across latent trait values or latent 'classes,' the factor variances and covariances are fixed at zero, and the only parameter differentiating classes is the factor mean. By constraining the item thresholds and factor loadings to be equal, we tested whether the disorder is 
being measured in the same way across classes. By constraining the factor covariance matrix to zero, we tested whether there are differences in severity between members with the same latent trait or latent 'class' value. The factor distribution is non-parametric because it is freely estimated or discretized into a finite number of values that give rise to quantitative differences in amounts of disorder limited in number of amounts to that of the number of latent trait values. Class membership is based on each individual's location on the factor, and this approach offers the most direct comparison to latent class analysis (i.e., values vs, classes).

\subsubsection{Latent Class/Qualitative Models of Liability}

Latent class analysis (LCA) identifies groups or classes of mutually exclusive individuals with the same patterns of item or symptom endorsement (Lazarsfeld \& Henry, 1968;

McCutcheon, 1987). Probable class membership of each individual is estimated based upon observed item endorsement patterns, and the items themselves are unrelated other than through their relationships to the classes (i.e., 'conditional independence'). Conceptually, all members within each class have the same underlying likelihood or liability toward endorsement of specific items. By comparing the fit of LCFAs and LCAs with the same number of class values, we tested whether discrete categories or finite gradations of severity better comprised liability.

\subsubsection{Hybrid Quantitative/Qualitative Models of Liability}

Factor mixture modeling (FMM) is a hybrid approach to conventional confirmatory factor analysis and LCA. Whereas LCA identifies classes of individuals endorsing specific patterns of symptoms and CFA elucidates latent dimensions underlying relationships between symptoms, FMM identifies classes of individuals with similar response patterns while simultaneously acknowledging the symptom structure of those response patterns. Imposition of a variety of measurement invariance constraints across latent classes can reveal whether the 
factors of the disorder are measured in the same way across classes (i.e., quantitative or qualitative differences across classes).

We initially tested for strong factorial invariance (Meredith, 1993) across classes of each FMM, the default for FMM in Mplus (Muthén \& Muthén, 1998-2012). In these models, we constrained item thresholds (i.e., intercepts in the regression of observed item response options on the factors), factor loadings, and the factor covariance matrix to be equal across classes, with only the factor means varying across classes. Here, the factors comprising the disorder are being measured in the same way across classes, the relationships between factors are the same across classes, and individual differences are accounted for by differences in severity on the factors (i.e., quantitative differences between classes).

Following identification of the best fitting of these models, we tested whether relaxation of specific constraints improved model fit in accordance with the steps described by Clark and colleagues (2013). The first model variant (FMM-1) also specified the factors to be measured the same way across classes by constraining item thresholds and factor loadings to be equal across classes, but both the factor covariance matrix and factor means were allowed to vary across classes. Here, the factors are a mixture of normal distributions located at different points on the factor distribution where each normal distribution comprises a class. The next set of model constraints imposed qualitative differences in the disorder such that the factors were not measured in the same way across classes. In the second model (FMM-2), we constrained factor loadings and the factor covariance matrix to be equal across classes, fixed the factor means to zero for model identification, and allowed the item thresholds to vary across classes. Here, the classes are based on differential responses to items across classes as opposed to the factors. In the third model variant (FMM-3), we constrained factor loadings to be equal across classes, fixed 
the factor means to zero, and allowed both the item thresholds and the factor covariance matrix to vary across classes. Here, the classes differ in range of severity given that factor variances were non-invariant across classes. Finally, in the fourth model (FMM-4), we allowed item thresholds, factor loadings, and the factor covariance matrix to vary across classes, with factor means fixed to zero. Here, the items evidence differential relationships with the factors across classes, and the classes differ in range of severity given that the factor variances were allowed to vary across classes.

Structural modeling analyses of the PCL and BDI-II were conducted in Mplus (version 7; Muthén \& Muthén, 1998-2012) using maximum likelihood estimation with standard errors robust (MLR) to non-normality. The PCL and BDI-II items were specified as ordered categorical (i.e., ordinal) variables given observed non-normal distributions. MLR allowed for the comparison of relative fit of non-nested models using information- theoretic fit indices. Missing data was treated with full information maximum likelihood. The Bayesian information criterion (BIC) favors models that balance absolute fit against increases in model complexity. Differences in BIC values of 10 indicate posterior odds of 150:1, or 'very strong' preference for the model of lesser BIC value (Raftery, 1995). Differences in BIC have been shown to better distinguish between non-nested LTMs and LCMs in simulation studies than other informationtheoretic indices computed in Mplus (Markon \& Krueger, 2006; Nylund, Asparouhov, \& Muthén, 2007). Model fit across time points was not directly assessed as these models are not directly comparable.

\section{Results}

\subsection{PTSD Latent Structure}


The fit of latent trait, latent class factor, latent class, and hybrid models of PTSD as measured by the PCL was compared using CFA, LCFA, LCA, and FMM, respectively. Fit statistics for PCL models are presented in Table 1.

\subsubsection{Latent Trait vs. Latent Class Models of Liability}

LTMs of PTSD fit better than LCMs at both time points. All four trait-dimensional CFA models fit better than any LCM, including the one-factor model. Comparison of LCFA models and LCA models with the same number of values and classes, respectively, also indicated preference for dimensional, finite gradations of severity over discrete categories of individuals with the same symptom endorsement patterns. The best-fitting of the LCMs was the three-class model both before and after deployment, suggesting that no new class emerged following combat exposure. Taken together, these findings suggest that dimensional conceptualizations of PTSD symptomatology better account for liability than categorical conceptualizations both before and after combat exposure.

\subsubsection{Hybrid Models of Liability}

The best-fitting model overall was the two-class, four-factor 'dysphoria' FMM at both time points. The two classes emerging in this model differed in terms of mean levels on each of the four factors, while the factor structure, item loadings, item thresholds, factor variances, and factor covariances were held equal across classes. Prior to deployment, the proportionally smaller latent class $(n=29,5.6 \%)$ evidenced greater mean levels on each of the four factors than the larger class $(n=487,94.4 \%)$, though the factors were measured the same way across classes. Following deployment, all four factor means were greater in magnitude in the smaller $(n=82$, $19.4 \%)$ than larger $(n=341,80.6 \%)$ latent class, again with measurement of the factors invariant across classes. This best-fitting two-class, four-factor FMM indicated that class membership 
reflected quantitative differences between classes in severity on the factors. We then tested whether the relaxation of measurement invariance constraints through allowance of quantitative or qualitative class differences improved model fit. Specifically, we compared whether specifying non-invariance across the two classes in the factor covariance matrix and factor means (FMM-1; quantitative), the item thresholds (FMM-2; qualitative), the item thresholds and the factor covariance matrix (FMM-3; qualitative), or the factor loadings, item thresholds, and the factor covariance matrix (FMM-4; qualitative) improved model fit. None of these models fit better than the two-class, four-factor FMM specifying strong measurement invariance. Model parameters for the best-fitting PCL mixture models are presented in Table 2.

\subsection{Depression Latent Structure}

EFA of the BDI-II at both pre-deployment and post-deployment informed specification of the two-factor models tested using CFA and FMM. The structure was consistent in terms of preferential item loadings on the Cognitive-Affective and Somatic-Vegetative factors reported by Beck et al. (1996), Dozois et al. (1998), and tested in meta-analysis (Model 3; Huang \& Chen, 2015). Specifically, items 1-3, 5-9, and 13-14 were specified to load on the Cognitive-Affective factor, and the remaining items served as indicators of the Somatic-Vegetative factor. Tested also was a three-factor model of Osman et al (1997) compared in meta-analysis (Model 7; Huang

\& Chen, 2015). Specifically, items 1-3, 5-10, and 14 were specified to load on the Negative Attitude factor, items 4, 12-13, 15, 17, and 19-20 marked the Performance Difficulty factor, and items 11, 16, 18, and 21 loaded on the Somatic Elements factor. The fit of CFA, LCFA, LCA, and FMM models of depression are presented in Table 3.

\subsubsection{Latent Trait vs. Latent Class Models of Liability}


Consistent with findings for PTSD, LTMs of depression fit better than LCMs at both time points. One- through three-factor trait-dimensional CFA models fit better than any LCM. LCFA and LCA model comparisons indicated trait dimensions with limited gradations of severity were preferred over discrete liability classes. The best-fitting of the LCMs was again the three-class model both before and after deployment. Dimensional conceptualizations of depression better account for liability than categorical conceptualizations both before and after combat exposure.

\subsubsection{Hybrid Models of Liability}

The best-fitting model for depression was the two-class, two-factor FMM specifying strong measurement invariance at both time points. The two classes emerging in this model differed in terms of mean levels on each of the two factors, while the factor structure, item loadings, item thresholds, factor variances, and factor covariances were held equal across classes. Prior to deployment, the proportionally smaller latent class $(n=16,3 \%)$ evidenced greater mean levels on both factors than the larger class $(n=499,97 \%)$. Following deployment, the larger latent class $(n=267,64 \%)$ evidenced greater mean levels on both factors than the smaller latent class $(n=152,36 \%)$. Relaxing the strong measurement invariance constraints of the two-class, two-factor model of depression by reducing them in the manner specified in FMM-1, FMM-2, FMM-3, and FMM-4 models above revealed that no alternative model fit better at either time point. Model parameters for the best-fitting BDI-II mixture model are presented in Table 4.

\section{Discussion}

The primary objective of this study was to examine whether PTSD represents a discrete diagnostic entity through evidence of qualitative differences between normative and pathological responses to combat-related trauma. We directly compared the fit of dimensional, categorical, and class-dimensional models of PTSD before and after deployment to test the hypothesis that 
the syndrome should qualitatively distinguish individuals distressed by traumatic events from those who are not. We suggested that qualitative differences could arise through emergence of a latent class following exposure that was not present prior to exposure, differences in measurement of the syndrome across affected and unaffected classes following exposure, and/or structural differences in the syndrome between time points. Our hypothesis was not supported, and similar findings in depression suggested parallels with a dimensional syndrome lacking etiological trauma-related assumptions.

\subsection{The Structure of PTSD}

We directly compared the fit of latent trait models, latent class models, and classdimensional models of PTSD. No LCM fit better than any parametric LTMs, and no LCM fit better than any of their non-parametric LTM counterparts. The finding that the latent structure of PTSD is better conceptualized as dimensional rather than categorical is consistent with taxometric analyses (Forbes et al., 2005; Ruscio et al., 2002), and points to the need for comparison of LTMs in studies employing latent class analysis. The four-factor 'dysphoria' CFA model fit better than the four-factor 'numbing' model, consistent with meta-analytic work (Yufik \& Simms, 2010). The three-class model was the best-fitting LCM, consistent with studies finding evidence for low, medium, and high disturbance classes (e.g., Ayer et al., 2011; Steenkamp et al., 2012). Finally, the two-class, four-factor 'dysphoria' mixture model fit better than the one-class, four-factor mixture model, consistent with prior research employing these methods (Naifeh et al., 2010; Shevlin \& Elklit, 2012). The best-fitting model overall was the class-dimensional hybrid model specifying the structure to be consistent with the 'dysphoria' model of PTSD and strong measurement invariance across two latent classes. Constraining the models across latent classes to have the same factor structure, item loadings, item thresholds, 
factor variances, and factor covariances, and allowing for differences in factor means indicated that the classes differed quantitatively in severity of symptom dimensions. Relaxing these constraints to allow for qualitative differences between classes did not improve model fit.

The prospective nature of the study's design allowed us to examine whether a distinctive class of individuals with PTSD would emerge following combat exposure as the assumption of specific etiology might suggest. The hypothesis that a qualitatively distinctive class would emerge following combat exposure was not supported. Assuming the inextricable linkage between the traumatic event(s) and subsequent symptom expression in the definition of PTSD, the latent structure should have looked markedly different prior to combat exposure than following combat exposure. Indeed this was not the case and a distinctive structure did not emerge in soldiers post-deployment. Further, the hypothesis that the symptom dimensions should qualitatively distinguish affected from unaffected classes following deployment was also not supported. The two-class, four-factor mixture model fit best at both time points, and only quantitative differences in severity on the four factors distinguished them at both time points. At pre-deployment, a small latent class $(n=29,5.6 \%)$ evidenced higher mean levels on all four PCL factors (particularly Intrusions). At post-deployment, a somewhat larger latent class ( $\mathrm{n}=$ 82, 19.4\%) evidenced higher mean levels on all four PCL factors (particularly Avoidance). Prior to deployment, the two-class, four-factor PCL mixture model likely empirically identified a chronically distressed group of individuals whose symptoms were unrelated to deployment related stressors. Alternatively, PTSD symptomatology may not develop de novo after trauma exposure, in that this class was evident prior to combat exposure and the structure and measurement of the syndrome was equivalent across affected and unaffected classes both before and after combat exposure. The presence of a small group of distressed individuals prior to 
deployment could be due to pre-existing PTSD symptoms stemming from previous trauma exposure (see, however, Footnote 1). It is important to note, however, that results were inconsistent with different latent structures across the distressed and non-distressed classes at either pre- or post-deployment time points. This suggests, at both time points, that distressed individuals were showing symptom differences in degree, not in kind, and argues against a distinct syndrome tied to exposure to a traumatic event.

\subsection{The Structure of Depression}

Given no requirement for a precipitating stressor or etiological criterion in the definition of depression, and general consensus that depression is trait-dimensional (e.g., Ruscio \& Ruscio, 2000), the structure of the syndrome was not expected to be markedly different between predeployment and post-deployment assessments. Similar findings in the latent structures of PTSD and depression would not be expected if trauma exposure were conceptually essential and inessential in expression of symptomatology of the former and latter disorders, respectively. We found the same class-dimensional structural model fit best for depression both prior to combat exposure and following combat exposure: a hybrid two-class, two-factor, strong measurement invariance model with classes differing in severity of factor means. The factor mean differences were flipped in terms of their importance in predicting pre- and post-deployment class membership; there were greater class mean differences in the cognitive-affective factor at predeployment and the somatic-vegetative factor following deployment. However, as with PTSD, the structure of the syndrome was invariant across affected and unaffected classes at both time points, further calling into question the necessity of trauma exposure in the expression of either disorder.

\subsection{Implications for Specific Instantiations of Distress}


The heuristic value of PTSD as a disorder distinctive from extant DSM syndromes has been called into question (e.g., Rosen \& Lilienfeld, 2008). There is no doubt that combat-related trauma can be distressing, as the proportion of individuals in the 'affected' classes increased from pre- to post-deployment. However, the findings herein imply that stress responses of affected individuals differ from those of unaffected individuals in degree, not in kind, from less debilitating reactions to potentially traumatic events. PTSD and depression have been viewed as indicators of a broader distress dimension in multivariate models of comorbidity between internalizing syndromes (Cox, Clara, \& Enns, 2002; Slade \& Watson, 2006). The structure of PTSD and depression may be conceptually similar because each facet of each syndrome may be a marker of a broader general distress domain. Indeed, in our sample, the BDI-II predicted postdeployment SCID-II PTSD diagnoses above and beyond an empirically-derived optimal total PCL cut score (Arbisi et al., 2012). A bottom-up approach to delineation of the structure of internalizing syndromes has been undertaken through development of the Inventory of Depression and Anxiety Symptoms (IDAS, IDAS-II; Watson et al., 2007; Watson et al., 2008; Watson et al., 2012). The structure of the IDAS yielded a general factor marked by Dysphoria accounting for approximately $90 \%$ of the variance in the domain (Watson et al., 2007). The IDAS-II Traumatic Intrusions and Traumatic Avoidance scales evidence strong convergence with PCL Intrusions $(r=.76)$ and PCL Avoidance $(r=.76)$ scales (Watson et al., 2012). Notably, the Traumatic Intrusions and Traumatic Avoidance IDAS scales loaded appreciably on the Distress factor of the IDAS-II, as did scales covering similar content to those of the BDI-II factors. Taken together, these findings suggest that PCL and BDI-II factors may be elements or facets of a broader internalizing domain (cf., Krueger, 1999; Wolf et al., 2014) that mark a general distress factor of this domain to varying degrees. The lack of inherent structural change 
or emergence of a distinctive PTSD class following combat exposure suggests that DSM-IV symptoms as measured by the PCL may do little more to delineate individuals with a unique trauma-related syndrome than to help distinguish between individuals high and low in general distress.

The implications for discretizing PTSD without clear delineation from normative stress responses may be deleterious to both investigations of the pathogenesis of the disorder and forensic evaluation of the syndrome's consequences. If PTSD represents one instantiation of a broader distress domain in structural models of psychopathology, then by definition it shares a great deal of variance with other disorders in this domain. Latent variable modeling approaches to studying risk factor specificity have shown that general internalizing and externalizing propensities account for gender differences in prevalence of (Kramer, Krueger, \& Hicks, 2008), and emergence of comorbidity between (Kessler et al., 2011), individual disorders. Acknowledging the covariance with other distress-related syndromes through prediction of the unique variance in PTSD symptomatology after taking this shared variance into account may better delineate general versus specific risk factors for the disorder. In a similar vein, clinical differentiation of PTSD from other distress disorders is complicated both by the endorsement of symptoms by those without significant trauma exposure (i.e., pre-deployment) and by quantitative (i.e., only severity) distinctions between affected and unaffected individuals. Since binary classification of PTSD has far-reaching consequences for disability claims, clinicians would be better served by criteria that qualitatively distinguish pathological from normative responses to trauma. For example, there is evidence that dissociative symptoms (i.e., flashbacks, depersonalization, and derealization) may qualitatively distinguish between severity classes showing otherwise largely parallel PTSD symptom endorsement profiles (Wolf et al., 2012). 
Further work of this kind identifying symptoms that qualitatively differentiate pathological from normative responses to stressors would facilitate demarcation of PTSD from other distress disorders, yielding greater confidence in high stakes evaluations.

\subsection{Limitations}

The findings of this study should be viewed in light of several limitations. First, the latent structures of the PCL and BDI-II were investigated in a National Guard sample in which only subsets of the sample evidenced clinically significant pre- and post-deployment pathology. Conversely, including in the sample individuals who did not screen positive for PTSD may also be considered a strength of the design in that it allowed for examination of measurement invariance across affected and unaffected classes. Second, the pre-deployment sample undoubtedly comprised some individuals who were previously exposed to some form of trauma (see Footnote 1). Regardless, the classes that emerged prior to deployment did not differ from the rest of the sample in terms of measurement of the syndromes. Third, the samples differed in size at pre- and post-deployment given attrition from the study. It is possible that the proportion of individuals in affected classes may have been different if all participants completed both waves of assessment. Finally, our findings contrast with a recent study finding factorial variance (i.e., differences in item thresholds) of a one-factor model of PTSD symptoms before and after deployment (Lommen, van de Schoot, \& Englehard, 2014). The analytic approach did not take into account the multi-factorial structure of the symptoms, however. Despite these limitations, our findings point to the need to more carefully examine whether the sequelae of trauma articulated in the DSM PTSD criteria adequately differentiate individuals with a trauma-specific diagnostic syndrome from those exhibiting a general stress reaction unrelated to an index trauma.

\section{Conclusions}


A failure to find a change in the structure or measurement of PTSD symptoms in response to the application of a stressor suggests that PTSD is not distinct from general distress. There are two possible explanations for this failure to find the expected change in structure (rather than simply level) of PTSD symptoms following a combat deployment. First, it is possible that current self-report inventories of PTSD symptoms are so saturated in general distress that the more unique (and presumably trauma related) aspects of the disorder failed to emerge in the analyses. Given that the self-report measures are essentially restatements of DSM symptom criteria, this could have implications for the disorder itself. This leads to a second more serious implication, which is that the symptoms of PTSD may not in themselves represent unique reactions to a unique class of events (i.e., Criterion A traumatic stressors). In other words, PTSD symptoms may be symptoms of general distress that tend to go up when one is exposed to stressors, such as Criterion A events, but that can be present, to greater or lesser extent, without such stressors ever having occurred. Such an interpretation calls into question the core assumptions of the PTSD diagnosis, and analyses of the current symptoms of PTSD in a similar manner could perhaps serve to refine the syndrome in future DSM iterations. 


\section{Footnote}

${ }^{1}$ The pre-deployment sample included 29/522 Soldiers who reported a prior Operation Iraqi Freedom (OIF) or Operation Enduring Freedom (OEF) deployment. All pre-deployment PTSD Checklist (PCL) analyses of latent trait models, latent class models, and factor mixture models reported in Table 2 were re-run with exclusion of these 29 cases. The same two-class, fourfactor 'dysphoria' model with strong measurement invariance across classes was the best-fitting model overall. 


\section{References}

American Psychiatric Association. (1987). Diagnostic and statistical manual of mental disorders (3rd ed., revised). Washington, DC: Author.

American Psychiatric Association. (1994). Diagnostic and statistical manual of mental disorders (4th ed.). Washington, DC: Author.

American Psychiatric Association. (2013). Diagnostic and statistical manual of mental disorders (5th ed.). Washington, DC: Author.

Arbisi, P. A., Kaler, M. E., Kehle-Forbes, S. M., Erbes, C. R., Polusny, M. A., \& Thuras, P. (2012). The predictive validity of the PTSD Checklist in a nonclinical sample of combatexposed National Guard troops. Psychological Assessment, 24(4), 1034-1040. doi: 10.1037/a0028014

Armour, C., Contractor, A., Elhai, J. D., Stringer, M., Lyle, G., Forbes, D., \& Richardson, J. D. (2015). Identifying latent profiles of posttraumatic stress and major depression symptoms in Canadian veterans: Exploring differences across profiles in health related functioning. Psychiatry Research, 228, 1-7. doi: 10.1016/j.psychres.2015.03.011

Arnau, R. C., Meagher, M. W., Norris, M. P., \& Bramson, R. (2001). Psychometric evaluation of the Beck Depression Inventory-II with primary care medical patients. Health Psychology, 20(2), 112-119. doi: 10.1037/0278-6133.20.2.112

Ayer, L., Danielson, C. K., Amstadter, A. B., Ruggiero, K., Saunders, B., \& Kilpatrick, D. (2011). Latent classes of adolescent posttraumatic stress disorder predict functioning and disorder after 1 year. Journal of the American Academy of Adolescent Psychiatry, 50(4), 364-375. doi:10.1016/j.jaac.2011.01.004 
Beck, A. T., Steer, R. A., \& Brown, G. K. (1996). Manual for the Beck Depression Inventory-II. San Antonio, TX: Psychological Corporation.

Bodkin, J. A., Pope, H. G., Detke, M. J., \& Hudson, J. I. (2007). Is PTSD caused by traumatic stress? Journal of Anxiety Disorders, 21, 176-182. doi:10.1016/j.janxdis.2006.09.004

Breslau, N., Reboussin, B. A., Anthony, J. C., \& Storr, C. L. (2005). The structure of posttraumatic stress disorder: Latent class analysis in 2 community samples. Archives of General Psychiatry, 62, 1343-1351. doi:10.1001/archpsyc.62.12.1343

Clark, S. L., Muthén, B., Kaprio, J., D’Onofrio, B. M., Viken, R., \& Rose, R. J. (2013). Models and strategies for factor mixture analysis: An example concerning the structure underlying psychological disorders. Structural Equation Modeling, 20(4), 1-29. doi:10.1080/10705511.2013.824786

Contractor, A. A., Elhai, J. D., Fine, T. H., Tamburrino, M. B., Cohen, G., Shirley, E., Chan, P. K., Liberzon, I., Galea, S., \& Calabrese, J. R. (2015). Latent profile analyses of posttraumatic stress disorder, depression, and generalized anxiety disorder symptoms in trauma-exposed soldiers. Journal of Psychiatric Research, 68, 19-26. doi:10.1016/j.jpsychires.2015.05.014

Cox, B. J., Clara, I. P., \& Enns, M. W. (2002). Posttraumatic stress disorder and the structure of common mental disorders. Depression and Anxiety, 15(4), 168-171. doi:10.1002/da.10052

Dozois, D. J. A., Dobson, K. S., \& Ahnberg, J. L. (1998). A psychometric evaluation of the Beck Depression Inventory-II. Psychological Assessment, 10(2), 83-89. doi: 10.1037/10403590.10 .2 .83 
Elhai, J. D., Biehn, T. L., Armour, C., Klopper, J. J., Frueh, B. C., \& Palmieri, P. A. (2011). Evidence for a unique PTSD construct represented by PTSD's D1-D3 symptoms. Journal of Anxiety Disorders, 25(3), 340-345. doi: 10.1016/j.janxdis.2010.10.007

Elhai, J. D., Engdahl, R. M., Palmieri, P. A., Naifeh, J. A., Schweinle, A., \& Jacobs, G. A. (2009). Assessing posttraumatic stress disorder with or without reference to a single, worst traumatic event: Examining differences in factor structure. Psychological Assessment, 21(4), 629-634. doi:10.1037/a0016677

Elhai, J. D., \& Palmieri, P. A. (2011). The factor structure of posttraumatic stress disorder: A literature update, critique of methodology, and agenda for future research. Journal of Anxiety Disorders, 25, 849-854. doi:10.1016/j.janxdis.2011.04.007

Erwin, B. A., Heimberg, R. G., Marx, B. P., \& Franklin, M. E. (2006). Traumatic and socially stressful life events among persons with social anxiety disorder. Anxiety Disorders, 20, 896-914. doi:10.1016/j.janxdis.2005.05.006

Forbes, D., Haslam, N., Williams, B. J., \& Creamer, M. (2005). Testing the latent structure of posttraumatic stress disorder: A taxometric study of combat veterans. Journal of Traumatic Stress, 18, 647-656. doi:10.1002/jts.20073

Gold, S. D., Marx, B. P., Soler-Baillo, J. M., \& Sloan, D. M. (2005). Is life stress more traumatic than traumatic stress? Journal of Anxiety Disorders, 19, 687-698. doi:10.1016/j.janxdis.2004.06.002

Gootzeit, J., \& Markon, K. E. (2011). Factors of PTSD: Differential specificity and external correlates. Clinical Psychology Review, 31, 993-1003. doi:10.1016/j.cpr.2011.06.005 Huang, C., \& Chen, J-H. (2015). Meta-analysis of the factor structures of the Beck Depression Inventory-II. Assessment, 22(4), 459-472. doi: 10.1177/1073191114548873 
Kessler, R. C., Cox, B. J., Green, J. G., Ormel, J., McLaughlin, K. A., Merikangas, K. R., Petukhova, M., Pine, D. S., Russo, L. J., Swendsen, J., Wittchen, H-U., \& Zaslavsky, A. M. (2011). The effects of latent variables in the development of comorbidity among common mental disorders. Depression and Anxiety, 28(1), 29-39. doi: 10.1002/da.20760

King, D. W., Leskin, G. A., King, L. A., \& Weathers, F. W. (1998). Confirmatory factor analysis of the Clinician-Administered PTSD Scale: Evidence for the dimensionality of posttraumatic stress disorder. Psychological Assessment, 10, 90-96. doi: 10.1037/10403590.10 .2 .90

Kramer, M. D., Krueger, R. F., \& Hicks, B. M. (2008). The role of latent internalizing and externalizing liability factors in accounting for gender differences in the prevalence of common psychopathological syndromes. Psychological Medicine, 38(1), 51-61. doi: http://dx.doi.org/10.1017/S0033291707001572

Krueger, R. F. (1999). The structure of common mental disorders. Archives of General Psychiatry, 56(10), 921-926. doi:10.1001/archpsyc.56.10.921

Lazarsfeld, P. F., and Henry, N. W. (1968). Latent Structure Analysis. Boston: Houghton Mifflin.

Lommen, M. J. J., van de Schoot, R., \& Englehard, I. M. (2014). The experience of traumatic events disrupts the measurement invariance of a posttraumatic stress scale. Frontiers in Psychology.

Lubke, G. H., \& Muthén, B. (2005). Investigating population heterogeneity with factor mixture models. Psychological Methods, 10(1), 21-39. doi: 10.1037/1082-989X.10.1.21 
Markon, K. E., \& Krueger, R. F. (2006). Information-theoretic latent distribution modeling: Distinguishing discrete and continuous latent variable models. Psychological Methods, 3, 228-243. doi: 10.1037/1082-989X.11.3.228

McCutcheon, A. C. (1987). Latent Class Analysis. Beverly Hills: Sage Publications.

Meehl, P. E. (1992). Factors and taxa, traits and types, differences of degree and differences in kind. Journal of Personality, 60, 117-174. doi: 10.1111/j.1467-6494.1992.tb00269.x

Meehl, P. E. (1995). Bootstraps taxometrics: Solving the classification problem in psychopathology. American Psychologist, 50, 266-275. doi: 10.1037/0003066X.50.4.266

Meis, L. A., Erbes, C. R., Kaler, M. E., Arbisi, P. A., \& Polusny, M. A. (2011). The structure of PTSD among two cohorts of returning Soldiers: Before, During, and Following deployment to Iraq. Journal of Abnormal Psychology, 120(4), 807-818. doi: $10.1037 / \mathrm{a} 0023976$

Meredith W. (1993). Measurement invariance, factor analysis and factorial invariance. Psychometrika, 58, 525-543.

Muthén, L. K., \& Muthén, B. O. (1998-2012). Mplus User's Guide. Sixth Edition. Los Angeles, CA: Muthén \& Muthén.

Naifeh, J. A., Richardson, J. D., Del Ben, K. S., \& Elhai, J. D. (2010). Heterogeneity in the latent structure of PTSD symptoms among Canadian veterans. Psychological Assessment, 22, 666-674. doi: 10.1037/a0019783

Nylund, K. L., Asparouhov, T., \& Muthén, B. O. (2007). Deciding on the number of classes in latent class analysis and growth mixture modeling: A Monte Carlo simulation study. Structural Equation Modeling, 14(4), 535-569. 
Osman, A., Downs, W. R., Barrios, F. X., Kopper, B. A., Gutierrez, P. M., \& Chiros, C. E. (1997). Factor structure and psychometric characteristics of the Beck Depression Inventory-II. Journal of Psychopathology and Behavioral Assessment, 19, 359-376.

Polusny, M. A., Erbes, C. R., Murdoch, M., Arbisi, P. A., Thuras, P., \& Rath, M. B. (2011). Prospective risk factors for new-onset posttraumatic stress disorder in National Guard soldiers deployed to Iraq. Psychological Medicine, 41(4), 687-698. doi: $10.1017 / \mathrm{S} 0033291710002047$

Raftery, A. E. (1995). Bayesian model selection in social research. Sociological Methodology, 25, 111-163.

Rosen, G. M. \& Lilienfeld, S. O. (2008). Posttraumatic stress disorder: An empirical evaluation of core assumptions. Clinical Psychology Review, 28, 837-868. doi:10.1016/j.cpr.2007.12.002

Ruscio, A. M., Ruscio, J., \& Keane, T. M. (2002). The latent structure of posttraumatic stress disorder: A taxometric investigation of reactions to extreme stress. Journal of Abnormal Psychology, 111, 290-301. doi: 10.1037/0021-843X.111.2.290

Ruscio, J. \& Ruscio, A. M. (2000). Informing the continuity controversy: A taxometric analysis of depression. Journal of Abnormal Psychology, 109(3), 473-487. doi: 10.1037/0021843X.109.3.473

Segal, D. L., Coolidge, F. L., Cahill, B. S., \& O'Riley, A. A. (2008). Psychometric properties of the Beck Depression Inventory-II among community-dwelling older adults. Behavior Modification, 32(1), 3-20. doi: 10.1177/0145445507303833 
Shafer, A. B. (2006). Meta-analysis of the factor structures of four depression questionnaires: Beck, CES-D, Hamilton, and Zung. Journal of Clinical Psychology, 62(1), 123-146. doi: 10.1002/jclp.20213

Shevlin, M., \& Elklit, A. (2012). The latent structure of posttraumatic stress disorder: Different models or different populations? Journal of Abnormal Psychology, 121(3), 610-615.

Simms, L. J., Watson, D., \& Doebbeling, B. N. (2002). Confirmatory factor analyses of posttraumatic stress symptoms in deployed and nondeployed veterans of the Gulf war. Journal of Abnormal Psychology, 111(4), 637-647. doi: 10.1037/0021-843X.111.4.637

Simons, D., \& Silveira,W. R. (1994). Posttraumatic stress disorder in children after television programmes. British Medical Journal, 308, 38-390.

Slade T., \& Watson, D. (2006). The structure of common DSM-IV and ICD-10 mental disorders in the Australian general population. Psychological Medicine, 36, 1593-1600. doi: $10.1017 / \mathrm{S} 0033291706008452$

Solomon, S. D., \& Canino, G. J. (1990). Appropriateness of the DSM-III-R criteria for posttraumatic stress disorder. Comprehensive Psychiatry, 31, 227-237. doi: 10.1016/0010-440X(90)90006-E

Steenkamp, M. M., Nickerson, A., Maguen, S., Dickstein, B. D., Nash, W. P., \& Litz, B. T. (2012). Latent classes of PTSD symptoms in Vietnam veterans. Behavior Modification, 36(6), 857-874. doi: 10.1177/0145445512450908

Waller, N. G., \& Meehl, P. E. (1998). Multivariate taxometric procedures: Distinguishing types from continua. Newbury Park, CA: Sage.

Watson, D., O’Hara, M. W., Chmielewski, M., McDade-Montez, E. A., Koffel, E., Naragon, K., \& Stuart, S. (2008). Further validation of the IDAS: Evidence of convergent, 
discriminant, criterion, and incremental validity. Psychological Assessment, 20, 248-259. doi: $10.1037 / \mathrm{a} 0012570$

Watson, D., O'Hara, M. W., Naragon-Gainey, K., Koffel, E., Chmielewski, M., Kotov, R., Stasik, S. M., \& Ruggero, C. J. (2012). Development and validation of new anxiety and bipolar symptom scales for an expanded version of the IDAS (the IDAS-II). Assessment, 19(4), 399-420. doi: 10.1177/1073191112449857

Watson, D., O'Hara, M., Simms, L. J., Kotov, R., Chmielewski, M., McDade-Montez, E. A., Gamez, W., \& Stuart, S. (2007). Development and validation of the inventory of depression and anxiety symptoms (IDAS). Psychological Assessment, 19(3), 253-268. doi: $10.1037 / 1040-3590.19 .3 .253$

Weathers, F.W., Litz, B.T., Herman, D.S., Huska, J.A., \& Keane, T.M. (1993). The PTSD Checklist (PCL): reliability, validity, and diagnostic utility. Paper presented at the Annual Meeting of the International Society for Traumatic Stress Studies, San Antonio, TX, October 1993.

Wolf, E. J., Miller, M. W., Kilpatrick, D., Resnick, H. S., Badour, C. L., Marx, B. P., Keane, T. M., Rosen, R. C., \& Friedman, M. J. (2014). ICD-11 Complex PTSD in U.S. National and Veteran Samples: Prevalence and structural associations with PTSD. Clinical Psychological Science, online ahead of print. doi: 10.1177/2167702614545480

Wolf, E. J., Miller, M. W., Reardon, A. F., Ryabchenko, K. A., Castillo, D., \& Freund, R. (2012). A latent class analysis of dissociation and posttraumatic stress disorder: Evidence for a dissociative subtype. Archives of General Psychiatry, 69(7), 698-705. doi: 10.1001/archgenpsychiatry.2011.1574 
Yufik, T., \& Simms, L. J. (2010). A meta-analytic investigation of the structure of posttraumatic stress disorder symptoms. Journal of Abnormal Psychology, 119, 764-776. doi: $10.1037 / \mathrm{a} 0020981$ 
Table 1

Fit statistics of latent trait, latent class, and hybrid models of the PCL

\begin{tabular}{|c|c|c|c|c|c|c|}
\hline & \multicolumn{3}{|c|}{ Pre-Deployment } & \multicolumn{3}{|c|}{ Post-Deployment } \\
\hline & $\underline{L L}$ & $\underline{k}$ & BIC & $\underline{L L}$ & $\underline{k}$ & BIC \\
\hline \multicolumn{7}{|c|}{ Latent Trait Models } \\
\hline \multicolumn{7}{|c|}{$C F A$} \\
\hline 1-f & $-7,143$ & 85 & 14,817 & $-7,968$ & 85 & 16,449 \\
\hline $3-f$ & $-7,087$ & 88 & 14,724 & $-7,837$ & 88 & 16,205 \\
\hline $4-f_{N}$ & $-7,001$ & 91 & 14,571 & $-7,762$ & 91 & 16,074 \\
\hline $4-f_{D}$ & $-6,988$ & 91 & 14,545 & $-7,709$ & 91 & 15,969 \\
\hline $5-\mathrm{f}$ & $-6,979$ & 95 & 14,551 & $-7,702$ & 95 & 15,979 \\
\hline \multicolumn{7}{|l|}{ LCFA } \\
\hline 2 -value & $-7,547$ & 86 & 15,632 & $-8,445$ & 86 & 17,409 \\
\hline 3 -value & $-7,257$ & 88 & 15,063 & $-8,174$ & 88 & 16,881 \\
\hline 4-value & $-7,178$ & 90 & 14,917 & $-8,042$ & 90 & 16,628 \\
\hline 5 -value & $-7,141$ & 92 & 14,856 & $-8,015$ & 92 & 16,587 \\
\hline 6-value & $-7,130$ & 94 & 14,846 & $-7,978$ & 94 & 16,525 \\
\hline \multicolumn{7}{|c|}{ Latent Class Models } \\
\hline $2-\mathrm{c}$ & $-7,472$ & 137 & 15,800 & $-8,391$ & 137 & 17,611 \\
\hline $3-\mathrm{c}$ & $-7,160$ & 206 & 15,607 & $-8,101$ & 206 & 17,448 \\
\hline $4-c$ & $-7,035$ & 275 & 15,788 & $-7,924$ & 275 & 17,511 \\
\hline $5-\mathrm{c}$ & $-6,927$ & 344 & 16,002 & $-7,796$ & 344 & 17,672 \\
\hline 6-c & $-6,845$ & 413 & 16,269 & $-7,679$ & 413 & 17,856 \\
\hline \multicolumn{7}{|c|}{ Factor Mixture Models } \\
\hline 2-c, 1-f & $-7,140$ & 87 & 14,823 & $-7,968$ & 87 & 16,461 \\
\hline $3-c, 1-f$ & $-7,130$ & 89 & 14,815 & $-7,965$ & 89 & 16,469 \\
\hline $4-c, 1-f$ & $-7,129$ & 91 & 14,827 & $-7,962$ & 91 & 16,474 \\
\hline $2-c, 3-f$ & $-7,046$ & 92 & 14,666 & $-7,820$ & 92 & 16,197 \\
\hline $3-c, 3-f$ & $-7,048$ & 96 & 14,695 & $-7,808$ & 96 & 16,196 \\
\hline $4-c, 3-f$ & $-7,028$ & 100 & 14,680 & $-7,806$ & 100 & 16,216 \\
\hline $2-c, 4-f_{D}$ & $-6,948$ & 96 & 14,497 & $-7,685$ & 96 & 15,950 \\
\hline FMM-1 & $-7,280$ & 90 & 15,123 & $-8,072$ & 90 & 16,689 \\
\hline FMM-2 & $-6,866$ & 160 & 14,732 & $-7,574$ & 160 & 16,115 \\
\hline FMM-3 & $-6,850$ & 170 & 14,763 & $-7,603$ & 170 & 16,234 \\
\hline FMM-4 & $-6,852$ & 191 & 14,896 & $-7,558$ & 191 & 16,270 \\
\hline $3-c, 4-f_{D}$ & $-6,397$ & 101 & 14,504 & $-7,680$ & 101 & 15,971 \\
\hline $4-c, 4-f_{D}$ & $-6,936$ & 106 & 14,535 & $-7,666$ & 106 & 15,974 \\
\hline
\end{tabular}

Note. f: factor(s). c: class(es). LL: log-likelihood. $k$ : number of parameters. BIC: Bayesian information criterion. Subscripts: ${ }_{\mathrm{N}}$ Numbing model, ${ }_{\mathrm{D}}$ Dysphoria model. 
Table 2

PCL four-factor latent trait and two-class, four-factor mixture model standardized parameters

\begin{tabular}{|c|c|c|c|c|c|c|}
\hline & \multicolumn{3}{|c|}{ Pre-Deployment } & \multicolumn{3}{|c|}{ Post-Deployment } \\
\hline & \multirow[t]{2}{*}{ 4- $f_{D}$} & \multicolumn{2}{|c|}{$2-c, 4-f_{D}$} & \multirow[t]{2}{*}{$4-f_{D}$} & \multicolumn{2}{|c|}{ 2-c, 4- $f_{D}$} \\
\hline & & c1 & $\mathrm{c} 2$ & & $\mathrm{c} 1$ & $\mathrm{c} 2$ \\
\hline \multicolumn{7}{|l|}{ f1 } \\
\hline 1. Memories & .86 & .74 & .74 & .87 & .87 & .87 \\
\hline 2. Nightmares & .86 & .73 & .73 & .81 & .80 & .80 \\
\hline 3. Flashbacks & .89 & .75 & .75 & .83 & .79 & .79 \\
\hline 4. Upset & .89 & .78 & .78 & .85 & .82 & .82 \\
\hline 5. Physio & .84 & .70 & .70 & .83 & .80 & .80 \\
\hline \multicolumn{7}{|l|}{$\mathrm{f} 2$} \\
\hline 6. Thoughts & .88 & .80 & .80 & .85 & .75 & .75 \\
\hline 7. Activities & .89 & .82 & .82 & .85 & .77 & .77 \\
\hline \multicolumn{7}{|l|}{ f3 } \\
\hline 8. Amnesia & .64 & .58 & .58 & .69 & .65 & .65 \\
\hline 9. Interest & .70 & .64 & .64 & .83 & .80 & .80 \\
\hline 10. Detached & .77 & .70 & .70 & .88 & .87 & .87 \\
\hline 11. Affect & .76 & .69 & .69 & .88 & .86 & .86 \\
\hline 12. Future & .75 & .69 & .69 & .74 & .70 & .70 \\
\hline 13. Insomnia & .72 & .65 & .65 & .67 & .64 & .64 \\
\hline 14. Irritability & .69 & .61 & .61 & .80 & .78 & .78 \\
\hline 15. Concentration & .79 & .73 & .73 & .79 & .77 & .77 \\
\hline \multicolumn{7}{|l|}{$\mathrm{f} 4$} \\
\hline 16. Vigilance & .79 & .77 & .77 & .88 & .84 & .84 \\
\hline 17. Startle & .90 & .90 & .90 & .92 & .95 & .95 \\
\hline \multicolumn{7}{|l|}{ factor means } \\
\hline f1 Intrusions & & 0.00 & 2.60 & & 0.00 & 0.73 \\
\hline f2 Avoidance & & 0.00 & 1.34 & & 0.00 & 2.12 \\
\hline f3 Dysphoria & & 0.00 & 1.54 & & 0.00 & 0.86 \\
\hline f4 Hyperarousal & & 0.00 & 1.04 & & 0.00 & 0.56 \\
\hline
\end{tabular}

Note. f: factor(s). c: class(es). Subscript: ${ }_{\mathrm{D}}$ Dysphoria model. Factor means were fixed at zero in the first class and estimated in the second class, representing standardized differences between classes. 
Table 3.

Fit statistics of latent trait, latent class, and hybrid models of the BDI-II

\begin{tabular}{|c|c|c|c|c|c|c|}
\hline & \multicolumn{3}{|c|}{ Pre-Deployment } & \multicolumn{3}{|c|}{ Post-Deployment } \\
\hline & $\underline{L L}$ & $\underline{k}$ & $\underline{\mathrm{BIC}}$ & $\underline{L L}$ & $\underline{k}$ & BIC \\
\hline \multicolumn{7}{|c|}{$\overline{\text { Latent Trait Models }}$} \\
\hline \multicolumn{7}{|c|}{$C F A$} \\
\hline $1-f$ & $-5,533$ & 80 & 11,565 & $-5,766$ & 83 & 12,035 \\
\hline $2-f$ & $-5,474$ & 81 & 11,454 & $-5,693$ & 84 & 11,894 \\
\hline $3-f$ & $-5,565$ & 82 & 11,642 & $-5,776$ & 85 & 12,065 \\
\hline \multicolumn{7}{|l|}{$L C F A$} \\
\hline 2-value & $-5,846$ & 81 & 12,197 & $-6,162$ & 84 & 12,833 \\
\hline 3-value & $-5,590$ & 83 & 11,698 & $-5,852$ & 86 & 12,224 \\
\hline 4-value & $-5,549$ & 85 & 11,630 & $-5,789$ & 88 & 12,109 \\
\hline 5-value & $-5,524$ & 87 & 11,590 & $-5,750$ & 90 & 12,045 \\
\hline 6-value & $-5,519$ & 89 & 11,594 & $-5,742$ & 92 & 12,041 \\
\hline \multicolumn{7}{|c|}{ Latent Class Models } \\
\hline $2-c$ & $-5,825$ & 119 & 12,394 & $-6,138$ & 125 & 13,033 \\
\hline $3-c$ & $-5,531$ & 179 & 12,181 & $-5,801$ & 188 & 12,739 \\
\hline 4-c & $-5,454$ & 239 & 12,401 & $-5,708$ & 251 & 12,934 \\
\hline $5-\mathrm{c}$ & $-5,397$ & 299 & 12,661 & $-5,634$ & 314 & 13,167 \\
\hline 6-c & $-5,350$ & 359 & 12,941 & $-5,580$ & 377 & 13,441 \\
\hline \multicolumn{7}{|c|}{ Factor Mixture Models } \\
\hline 2-c, $1-f$ & $-5,528$ & 82 & 11,568 & $-5,750$ & 85 & 12,014 \\
\hline 3-c, $1-f$ & $-5,524$ & 84 & 11,572 & $-5,739$ & 87 & 12,004 \\
\hline 4-c, 1-f & $-5,523$ & 86 & 11,583 & $-5,736$ & 89 & 12,011 \\
\hline $2-c, 2-f$ & $-5,463$ & 84 & 11,450 & $-5,681$ & 87 & 11,888 \\
\hline FMM-1 & $-5,483$ & 141 & 11,847 & $-5,705$ & 147 & 12,298 \\
\hline FMM-2 & $-5,391$ & 140 & 11,656 & $-5,610$ & 143 & 12,084 \\
\hline FMM-3 & $-5,401$ & 145 & 11,708 & $-5,627$ & 148 & 12,149 \\
\hline FMM-4 & $-5,396$ & 166 & 11,828 & $-5,567$ & 169 & 12,156 \\
\hline $3-c, 2-f$ & $-5,459$ & 87 & 11,461 & $-5,678$ & 90 & 11,900 \\
\hline 4-c, 2-f & $-5,460$ & 90 & 11,481 & $-5,674$ & 93 & 11,910 \\
\hline
\end{tabular}

Note. f: factor(s). c: class(es). LL: log-likelihood. $k$ : number of parameters. BIC: Bayesian information criterion. 
Table 4

BDI-II two-factor latent trait and two-class, two-factor mixture model standardized parameters

\begin{tabular}{|c|c|c|c|c|c|c|}
\hline & \multicolumn{3}{|c|}{ Pre-Deployment } & \multicolumn{3}{|c|}{ Post-Deployment } \\
\hline & \multirow[t]{2}{*}{$2-f$} & \multicolumn{2}{|c|}{$2-c, 2-f$} & \multirow[t]{2}{*}{$2-f$} & \multicolumn{2}{|c|}{$2-c, 2-f$} \\
\hline & & $\mathrm{c} 1$ & c2 & & $\mathrm{c} 1$ & $\mathrm{c} 2$ \\
\hline \multicolumn{7}{|l|}{ f1 } \\
\hline 1. Sadness & .85 & .80 & .80 & .81 & .78 & .78 \\
\hline 2. Pessimism & .71 & .66 & .66 & .77 & .74 & .74 \\
\hline 3. Failure & .75 & .72 & .72 & .81 & .77 & .77 \\
\hline 5. Guilt & .68 & .65 & .65 & .78 & .74 & .74 \\
\hline 6. Punishment & .68 & .64 & .64 & .76 & .73 & .73 \\
\hline 7. Self-Dislike & .85 & .82 & .82 & .86 & .83 & .83 \\
\hline 8. Self-Critical & .78 & .74 & .74 & .88 & .85 & .85 \\
\hline 9. Suicidal & .82 & .78 & .78 & .76 & .71 & .71 \\
\hline 13. Indecisive & .83 & .79 & .79 & .76 & .73 & .73 \\
\hline 14. Worthless & .85 & .83 & .83 & .92 & .90 & .90 \\
\hline \multicolumn{7}{|l|}{$\mathrm{f} 2$} \\
\hline 4. Pleasure & .78 & .75 & .75 & .88 & .82 & .82 \\
\hline 10. Crying & .68 & .64 & .64 & .61 & .49 & .49 \\
\hline 11. Agitation & .77 & .76 & .76 & .76 & .64 & .64 \\
\hline 12. Interest & .84 & .82 & .82 & .90 & .84 & .84 \\
\hline 15. Energy & .84 & .83 & .83 & .84 & .74 & .74 \\
\hline 16. Sleep & .69 & .67 & .67 & .66 & .53 & .53 \\
\hline 17. Irritability & .78 & .77 & .77 & .74 & .62 & .62 \\
\hline 18. Appetite & .66 & .63 & .63 & .68 & .57 & .57 \\
\hline 19. Concentration & .80 & .78 & .78 & .72 & .60 & .60 \\
\hline 20. Fatigue & .84 & .83 & .83 & .83 & .73 & .73 \\
\hline 21. Libido & .50 & .48 & .48 & .72 & .61 & .61 \\
\hline \multicolumn{7}{|l|}{ factor means } \\
\hline f1 Cog-Affective & & 0.00 & 1.31 & & 0.00 & 0.97 \\
\hline f2 Somatic-Veg & & 0.00 & 0.60 & & 0.00 & 1.90 \\
\hline
\end{tabular}

Note. f: factor(s). c: class(es). Factor means were fixed at zero in the first class and estimated in the second class, representing standardized differences between classes. 